\title{
Nutrients and environmental factors as regulators of gene expression
}

\author{
Eva Enmark and Jan-Åke Gustafsson
}

Departments of Biosciences and Medical Nutrition, Karolinska Institute, NOVUM, Huddinge, Sweden

\begin{abstract}
A bstract
Several nuclear receptors bind substances in the diet or metabolites of dietary substances. This makes nuclear receptors a fascinating link between nutrition, toxicology, endocrinology and molecular biology. Nuclear receptor ligands may broadly be divided into two groups. The first group consists of steroid and thyroid hormones, and compounds that constitute intermediates in different biosynthetic pathways in steroid hormone, sterol and bile acid biosynthesis. The second group consists of the very diverse array of compounds that, with no or minor modifications, directly originate from foods, drugs or environmental pollutants. This review summarizes recent results on nuclear receptors with an emphasis on those with ligands of dietary relevance. The finding that several dietary compounds regulate gene expression in the same manner as steroid hormones has provided tools to help unravel molecular secrets and mechanisms behind many diet-associated diseases such as diabetes and obesity.
\end{abstract}

Keywords: Cholesterol, fatty acid, nuclear receptor, orphan receptor, phyto-oestrogen, vitamin.

Received: February 15, 2002. Accepted February 19, 2002

\section{Introduction}

Nuclear receptors constitute a large group of transcription factors which are involved in many important biological processes, e.g. embryonic development, fertility and regulation of metabolism. Approximately 50 members of this protein family are known in mammals, of which around 28 have known ligands or activators. A measure of their importance is the interest these receptors attract from the pharmaceutical industry, as targets for drugs directed against diseases such as diabetes, cancer and hypercholesterolaemia.

Recently, complete nucleotide sequences have become available for a few representatives of the animal kingdom: Caenorhabditis elegans, a nematode, Drosophila melanogaster, an insect, and Homo sapiens, a mammal (1). It is thus possible to finalize the number of nuclear receptors in these species, and to concentrate future efforts on characterization of these genes and their role in, for example, human physiology.

The "founders" of the nuclear receptor gene family were cloned during the 1980s. These were receptors for steroid hormones as oestradiol-17 $\beta$, testosterone, progesterone, aldosterone and cortisol. In addition, it turned out that the receptors for thyroid hormone and vitamin A belong to the same gene family. Soon thereafter, many laboratories reported on the isolation of additional members of the same protein family, but for which no ligand had been identified and they were thus termed "orphan" receptors (2). Several of these receptors have subsequently been found to bind to substances in the diet or to metabolites of dietary substances. This makes nuclear receptors a fascinating link between nutrition, toxicology, endocrinology and molecular biology.

\section{Basic concepts}

To be able to understand the mechanisms of action of these proteins, one must be familiar with some basic facts about their structure and function at the molecular level.

The nuclear receptors are ligand-activated transcription factors that either up- or down-regulate transcription of specific target genes via binding to sites (response elements) in the promoter region upstream of each target gene, resulting in changes in the activity of the basic transcription machinery (3). These effects of nuclear receptors are mediated by coactivators or corepressors that form complexes with the receptors and that interact with the basic transcription machinery (4) (Fig. 1). 


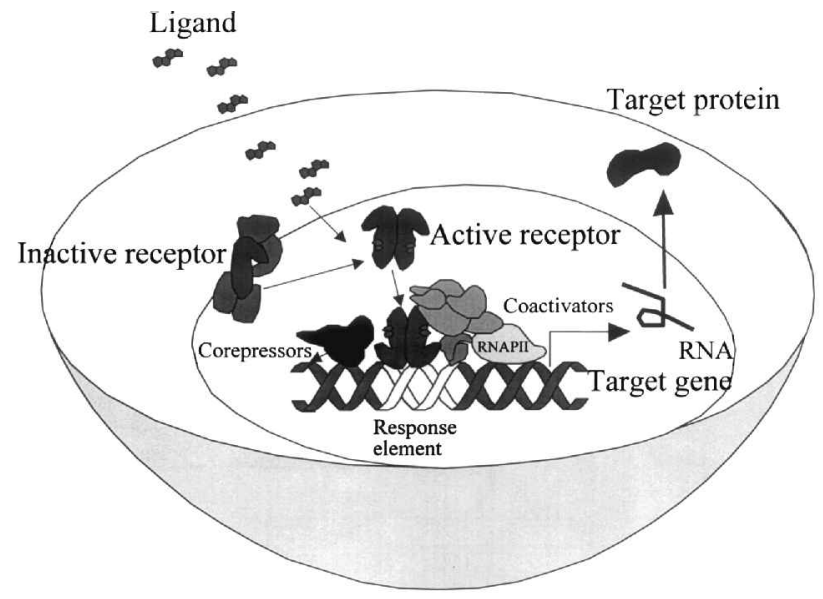

Fig. 1. Schematic model of the mechanism of action of nuclear receptors. RNA Polymerase II (RNAPII).

The most characteristic part of the nuclear receptors is a DNA-binding domain (DBD) comprised of two $\mathrm{Cys}_{2}-\mathrm{Cys}_{2}$ zinc-coordinating modules. Even the most distantly related nuclear receptors identified in mammalian species share at least $40 \%$ identity at the amino acid level in this domain.

The ability of a nuclear receptor to regulate gene expression is in many cases modulated by its binding of a ligand to a domain positioned carboxy-terminally to the DBD. This domain, the ligandbinding domain (LBD), also participates in homodimerization or heterodimerization with other LBDs, and in transcriptional regulation. Although the overall degree of sequence identity between different nuclear receptors is relatively low in the LBD, this part of the protein also contains regions that are conserved between most receptors.

Several receptors, for example the oestrogen and androgen receptors, may also be activated by ligand-independent mechanisms such as phosphorylation.

\section{Ligands in the diet}

Nuclear receptor ligands may largely be divided into two groups. The first group consists of steroid and thyroid hormones, and compounds that constitute intermediates in different biosynthetic pathways in steroid hormone, sterol and bile acid biosynthesis. The second group consists of the very diverse array of compounds that, with no or minor modifications, directly originate from foods, drugs or environmental pollutants. The distinction between these two groups is not absolutely strict, and there are several examples of nuclear receptors that may be activated by representatives of both groups, e.g. both by a steroid metabolite and by an environmental pollutant (Fig. 2).

\section{Cholesterol and steroid metabolites are nuclear receptor ligands}

Steroid hormones are all derived from cholesterol. Cholesterol has many physiological functions as well as pathophysiological effects (e.g. involvement in atherosclerosis). It is crucial as a precursor in

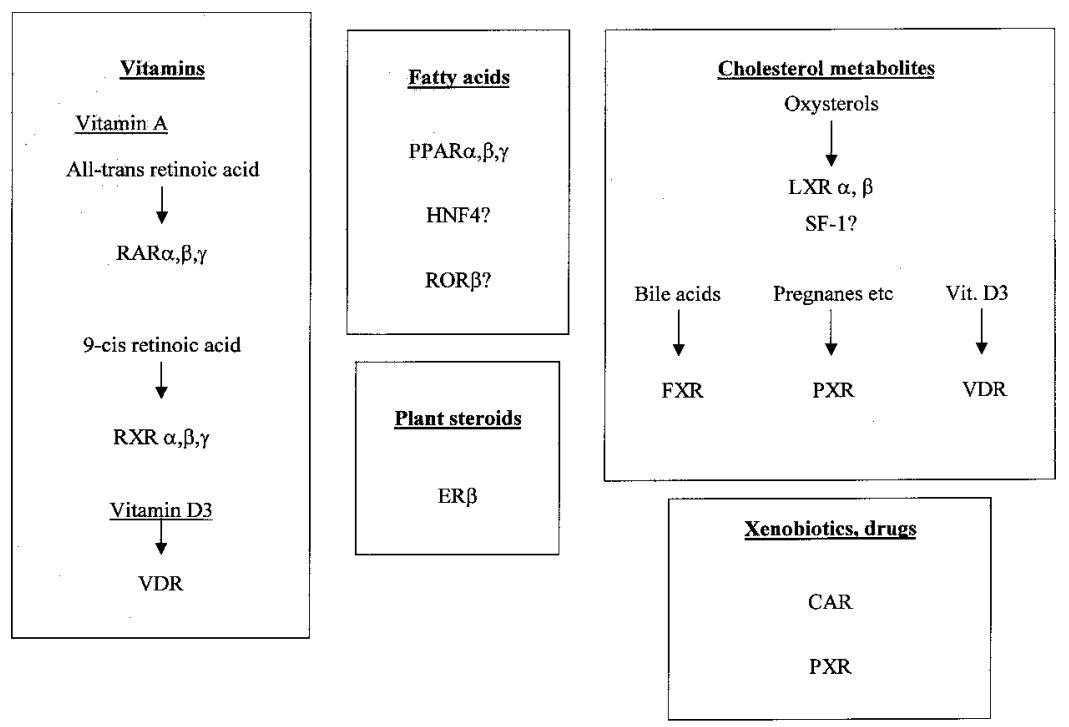

Fig. 2. Summary of receptors activated by dietary compounds. RAR: retinoic acid receptor; RXR: retinoic X receptor; VDR: vitamin D receptor; PPAR: peroxisome proliferator activated receptor; HNF: hepatic nuclear factor; ROR: retinoic acid receptor-like orphan receptor; ER: estrogen receptor; LXR: liver X receptor; SF: steroidogenic factor; FXR: X[Au: Define FXR?] receptor; PXR: pregnane X receptor; Vit. D3: vitamin $\mathrm{D}_{3}$; VDR: vitamin $\mathrm{D}$ receptor; CAR: constitutive androstane receptor. 
steroid hormone biosynthesis. The enzymes involved in cholesterol biosynthesis and further metabolism to steroid hormones and bile acids are carefully controlled in the organism, e.g. to avoid excessive accumulation of cholesterol in various tissues. Several nuclear receptors with ligands identified during recent years are involved in the regulation of these metabolic steps; often the ligands constitute intermediates in these pathways.

\section{$L X R$}

Formation of bile acids from cholesterol involves several hydroxylations. Two nuclear receptors, liver X receptor- $\alpha(\mathrm{LXR}-\alpha)$ and LXR- $\beta$ (NR1H3, NR1H2), have been shown to be activated by hydroxylated cholesterols, oxysterols. Recently, it was found that the naturally occurring oxysterols 24(S)-hydroxycholesterol, $22(R)$-hydroxycholesterol and 24(S),25-epoxycholesterol are potent ligands and activators of LXR- $\alpha$ and $-\beta$ (5-7). The most informative studies regarding the biological function of these nuclear receptors have been conducted in mice where either or both of these receptor genes have been inactivated. Feeding a diet containing high levels of cholesterol to mice carrying these mutations showed that the LXR- $\alpha$ knockout mice rapidly developed fatty liver due to defective clearance of cholesterol from this tissue (8), whereas inactivation of LXR- $\beta$, which has a very similar ligand-binding specificity to LXR- $\alpha$, does not lead to any apparent liver phenotype (9). It is not yet known whether there are genes that are uniquely regulated by only one of the receptor subtypes. Several examples have been published of genes that are regulated by both receptors, such as sterol regulatory element-binding protein-1c (SREBP-1c) (10), but in view of the striking differences in the phenotypes of the LXR- $\alpha$ and LXR- $\beta$ mouse strains, it is likely that specific LXR subtype target genes will be identified.

\section{FXR}

One nuclear receptor, Farnesoid $X$ Receptor (FXR; also termed BAR, NR1H4), is activated by physiological concentrations of chenodeoxycholic acid (11). FXR regulates the expression of the rate-limiting step in bile acid formation, cholesterol $7 \alpha$-hydroxylation, catalysed by the cytochrome P-450 isoform CYP7A1. As expected, this key gene in cholesterol homoeostasis is subject to a sophisticated regulation, being up-regulated by LXR- $\alpha$ (activated by a metabolite derived from one of the intermediates in cholesterol biosynthe- sis) and down-regulated by FXR (activated by a bile acid). In this way, formation of bile acids from cholesterol is regulated through both feedforward and feedback mechanisms (12). Whereas LXR- $\alpha$ appears to interact directly with the $5^{\prime}$ regulatory flank of CYP7A1, FXR first induces the orphan nuclear receptor short heterodimer partner (SHP, NR0B2), which acts as a repressor of CYP7A1 activity by inactivating the nuclear receptor LRH (FTF, NR5A2) which normally upregulates CYP7A1.

\section{$V D R$}

A third product, besides steroid hormones and bile acids, which is derived from cholesterol is vitamin $\mathrm{D}_{3}$. This physiologically extremely important compound with roles in skeletal and calcium homoeostasis, among others, acts through a member of the nuclear receptor family, the vitamin D receptor (VDR, NR1I1) (13). VDR regulates the expression of several genes encoding proteins participating in bone formation, e.g. the parathyroid receptor and osteocalcin (14). Interesting results have recently been published regarding newly developed synthetic vitamin D agonists and antagonists, based on the crystal structure of the vitamin $\mathrm{D}$ receptor (15). These may potentially have clinical uses in the treatment of, for example, prostate and breast cancer, traditionally not regarded as vitamin $\mathrm{D}$ targets.

\section{SF-I}

Another nuclear receptor, steroidogenic factor-1 (SF-1, NR5A1), has also been reported to be activated by hydroxylated cholesterol (16). This report has been questioned (17), but the receptor still deserves some attention. The main role of this receptor, as known so far, is in regulating the enzymes involved in steroid hormone biosynthesis, e.g. aromatase (CYP19), the enzyme responsible for converting oestradiol to testosterone. Structurally, SF-1 shows the highest sequence similarity to LRH1 (see above). A mouse SF-1 knockout has been created, and this strain has a phenotype remarkably similar to that of a form of hereditary adrenal hypoplasia (AHC). This disease has been genetically mapped to mutations in another unusual member of the nuclear receptor superfamily, DAX1 (NR0B1), which has been shown to act as a corepressor on SF-1 in a similar manner to SHP, a close relative of DAX1, which acts on LRH1 (18). 


\section{Vitamins and fat}

Dietary fat is one of the most important macronutrients for the growth of all animals, as a source of both energy and of hydrophobic components used in the synthesis of, for instance, membrane lipids. Genomic effects of fat may reflect a response to too much or too little fat in the diet, or to changes in the composition of the fat. Several fatty acid-regulated transcription factors have been identified in a wide variety of organisms ranging from bacteria to mammals (19). In mammals, these factors include several nuclear receptors. The peroxisome proliferator activated receptors (PPAR- $\alpha,-\delta$ and $-\gamma$ ), hepatic nuclear factor-4 (HNF-4) and retinoic acid receptor-like orphan receptor- $\beta$ (ROR- $\beta$ ) are known to be regulated by and/or bind fatty acid metabolites. Efforts have focused mainly on PPAR $-\gamma$ as a mediator of fatty acid effects on cell metabolism and differentiation, since PPAR- $\gamma$ is an important switch in the differentiation of preadipocytes to adipocytes, but it is likely that fatty acid-nuclear receptor interactions have important regulatory roles in many physiological contexts.

\section{PPAR}

All three PPAR subtypes have important roles in lipid metabolism. PPAR- $\alpha$ (NR1C1), the first PPAR to be cloned, is activated by, for example, eicosapentaenoic acid $(20: 5, n 3)$ and also, to a lesser extent, by a broad spectrum of other fatty acids $(20 a, b)$. PPAR $-\alpha$ may also be activated by an amazing variety of other compounds, including exogenous ligands such as fibrates (WY14, 643 and clofibrate) and plasticizers (phtalates) (21). It is expressed mainly in liver, heart, kidney and intestines, and a recently developed PPAR- $\alpha$ knockout mouse has provided important information about the function of this PPAR isoform. Studies of this mouse strain have shown that PPAR- $\alpha$ is involved in the regulation of several hepatic peroxisomal enzymes (acyl-CoA oxidase, bifunctional enzyme, thiolase) and microsomal enzymes, as well as certain apolipoproteins and fatty acid transport proteins (22).

PPAR- $\delta$ (also known as Nuc1, FAAR or PPAR$\beta, \mathrm{N} 1 \mathrm{C} 2$ ) is expressed in a broad range of tissues. Ligand-binding studies show that fatty acids and their metabolites are better ligands for this receptor than fibrates and other pharmacological substances (23). So far, no gene or pathway has been identified that is specifically regulated by PPAR- $\delta$.

PPAR $-\gamma(\mathrm{N} 1 \mathrm{C} 1)$ exists as two different splicing isoforms, PPAR- $\gamma_{1}$ and PPAR- $\gamma_{2}$, and is expressed in adipose tissue, spleen, retina, haematopoietic cells and epithelial cells in, for example, the colon (24). PPAR $-\gamma_{2}$ is the isoform that mainly participates in adipocyte differentiation (see above). This receptor is also involved in the induction of enzymes engaged in lipid storage. PPAR $-\gamma$ has been shown to bind specifically to a class of compounds termed thiazolidinediones (TZDs), such as troglitazone and pioglitazone (25). TZDs promote adipocyte differentiation and have antidiabetic effects. Troglitazone is used clinically to help to control blood glucose levels in patients with noninsulin-dependent diabetes (26). Other studies have shown that certain eicosanoids, e.g. 15-deoxy$\Delta^{12,14}$-prostaglandin $\mathrm{J} 2$, also bind to PPAR- $\gamma$, although it is questionable whether the concentrations needed are physiological $\left(K_{\mathrm{d}}\right.$ of $\left.2.5 \mu \mathrm{M}\right)(27)$.

Different studies have suggested that both PPAR- $\alpha$ and PPAR $-\gamma$ may play a role in inflammatory processes and, together with oxidized fatty acid metabolites, PPAR $-\gamma$ has also been proposed to play a role in the progression of atherosclerosis (28).

\section{$H N F-4$}

There are two mammalian subtypes of HNF-4, $\alpha$ (NR2A1) and $\gamma$ (R2A2), where HNF-4 $\alpha$ is expressed at much higher levels, predominantly but not exclusively in the liver. In 1998, it was reported that palmitoyl-CoA bound to $\mathrm{HNF}-4 \alpha$ at close to in vivo concentrations $\left(K_{\mathrm{d}} 2.6 \mu \mathrm{M}\right)(29)$. Although subsequent studies have challenged this report, it is possible that some fatty acid will eventually be identified as the true physiological ligand of HNF$4 \alpha$. HNF-4 regulates the transcription of many important genes, such as ApoCIII and L-PK in the liver (30). This receptor has an interesting link to human disease, namely maturity-onset diabetes of the young (MODY-1), a rare familial form of diabetes. Certain mutations in the HNF- $4 \alpha$ gene have been linked to this disease, and recently some models have been presented to explain the molecular mechanisms behind this connection (31, 32). HNF-4 seems to be a central regulator of glucose and lipid metabolism, and even patients carrying a heterozygous mutation in this gene have significantly altered levels of, for example, triglycerides and several apolipoproteins (33).

\section{$R O R$}

RORs (NR1F1, 2, 3) are a group of orphan receptors mainly expressed in different parts of the brain, where ROR- $\beta$ shows the highest degree of 
tissue specificity, being expressed in areas involved in the processing of sensory information, including the spinal cord, retina and pineal gland. An ROR$\beta$ knockout mouse strain has been produced, which exhibits a similar phenotype to the spontaneous mutation vacillans, with disorganized retinae, a "duck-like" gait and partial male infertility (34). Recently, Moras and co-workers (35) published a paper showing the LBD of ROR- $\beta$, bound to a stearate ligand. Since this particular compound is unable to activate the receptor in an in vitro assay, it is unlikely that it represents the physiological ligand to ROR- $\beta$, but it still may give an important clue to the nature of such a ligand.

\section{$R A R$ and $R X R$}

The retinoids are important factors in a number of tissues, such as the retina and the central nervous system. No fewer than six nuclear receptors are involved in mediating these functions, the retinoic acid receptors $\alpha, \beta$ and $\gamma$ (RAR- $\alpha,-\beta,-\gamma$, N1B1, 2 , 3 ) and retinoid $\mathrm{X}$ receptors $\alpha, \beta$ and $\gamma(\mathrm{RXR}-\alpha,-\beta$, $-\gamma, \mathrm{N} 2 \mathrm{~B} 1,2,3)$ (36).

The RXRs are fascinating proteins in their dual role of being both receptors with physiological ligands, as well as heterodimeric partners of a wide array of other nuclear receptors, such as Thyroid hormone receptor (TR), RAR, VDR and the PPARs (37). Several years ago it was shown that 9-cis retinoic acid, a stereoisomer of vitamin A, could activate RXR. Recently, other potentially physiological ligands to RXR have been suggested, e.g. docosahexaenoic acid and phytanic acid (38). The role of RXR as a partner of many receptors, thereby being involved in many biological processes, has resulted in rapid progress in the development of RXR-directed drugs; synthetic ligands for RXR, known as rexinoids, show promising results in the treatment of metabolic disorders such as diabetes and obesity (39).

\section{Plant steroids}

Several substances of plant origin present in the daily diet, in at least some cultures, serve as good ligands for the oestrogen receptors (ERs), and are therefore often referred to as phyto-oestrogens. This is, for example, true for genistein and coumestrol, two compounds present in soya, and zearalenone, which is an oestrogenic mycotoxin.

\section{O estrogen receptor $-\beta$}

In 1996, a novel subtype of the oestrogen receptor, ER- $\beta$ (NR3A2), was cloned (40). This receptor is expressed in several tissues where it could potentially encounter the plant oestrogens mentioned above, e.g. the epithelium of the small intestine and the colon. Several phyto-oestrogens have a higher affinity for ER- $\beta$ than for ER- $\alpha$ (41). It is thus likely that ER- $\beta$ plays an important role in mediating some of the effects that have been attributed to diets rich in phyto-oestrogens, such as soya products. Certain beneficial effects have been suggested in the context of menopausal symptoms and cardiovascular disease (42).

\section{Endocrine disruptors and other unnatural ligands}

It is poorly understood why so many compounds from the plant kingdom show affinity to mammalian sex hormone receptors. It was recently suggested (43) that this phenomenon is a reflection of the plant's defence against being eaten by different herbivores, by reducing the breeding capacity of these animals. As several of these substances have been shown to be beneficial to humans at lower concentrations, it is perhaps questionable whether this is a valid hypothesis.

This brings us to the subject of other substances that might serve as ligands to nuclear receptors and be consumed by humans.

\section{$P X R$}

The recently discovered pregnane $X$ receptor (PXR, NR1I2) plays a central role in the metabolism of many drugs. It is activated both by naturally occurring steroids such as pregnenolone and progesterone, and by synthetic glucocorticoids and antiglucocorticoids (44). Furthermore, it upregulates the cytochrome $\mathrm{P}-450$ isoform $3 \mathrm{~A} 4$ (CYP3A4), responsible for the oxidative metabolism of approximately $60 \%$ of all clinically used drugs (45). It is responsible for many interactions between drugs, where the intake of one drug may speed up the metabolism of another drug, which thereby may reach such low plasma levels as to become inactive in the patient.

One example of such interactions between drugs, where PXR may be involved, is the recent discovery that intake of St Johns wort, a mild seducing agent, lowers the effect of certain oral contraceptives, as St Johns wort contains hyperforin, a potent activator of PXR (46).

\section{CAR}

Constitutive androstane receptor (CAR, NR1I3) is an apparently constitutive transactivator, its activ- 
ity being inhibited by androstanes acting as inverse agonists (47). However, CAR transactivation is increased in the presence of 1,4-bis[2-(3,5-dichloropyridyloxy)]benzen e (TCPOBOP), the most potent known member of the phenobarbital-like class of CYP P-450-inducing agents (48). It has also been shown that a subset of xenobiotic ligands may activate both CAR and PXR, adding even further complexity to the topic of regulation of drug metabolism (49).

\section{Conclusion}

Nuclear receptors have turned out to be of interest not only to endocrinologists, pharmacologists and medicinal chemists, but also to toxicologists and nutritionists. Indeed, the insight that nutrients operate in similar ways to many hormones has meant a paradigm shift in nutrition and has helped to create a new and modern subdiscipline within nutrition, namely molecular nutrition. We now have tools to help to unravel the molecular secrets and mechanisms behind many diseases associated with diet, such as diabetes and obesity, and to obtain a more rational basis for giving dietary advice to healthy and diseased individuals. Nutrition is no longer a mainly phenomenological discipline but has qualified as a truly experimental science offering fantastic and exciting opportunities for young researchers eager to understand the molecular details of how diet affects our bodies.

\section{Acknowledgements}

This work was supported by grants from the Swedish Cancer Fund and from KaroBio AB.

\section{References}

1. Enmark E, Gustafsson JA: Comparing nuclear receptors in worms, flies and humans. Trends Pharmacol Sci 2001;22:611-5.

2. Giguere V: Orphan nuclear receptors: from gene to function. Endocr Rev 1999;20:689-725.

3. Gronemeyer H, Laudet V: Transcription factors 3: nuclear receptors. Protein Profile 1995;2:1173-308.

4. McKenna NJ, Xu J, Nawaz Z et al: Nuclear receptor coactivators: multiple enzymes, multiple complexes, multiple functions. J Steroid Biochem Mol Biol 1999;69:3-12.

5. Song C, Liao S: Cholestenoic acid is a naturally occurring ligand for liver $\mathrm{X}$ receptor alpha. Endocrinology 2000;141:418 0-4.

6. Janowski BA, Grogan MJ, Jones SA et al: Structural requirements of ligands for the oxysterol liver $\mathrm{X}$ recep- tors LXRalpha and LXRbeta. Proc Natl Acad Sci USA 1999;96:266-71.

7. Lehmann JM, Kliewer SA, Moore LB et al: Activation of the nuclear receptor LXR by oxysterols defines a new hormone response pathway. $J$ Biol Chem 1997;272:3137-40.

8. Peet DJ, Turley SD, Ma W et al: Cholesterol and bile acid metabolism are impaired in mice lacking the nuclear oxysterol receptor LXR alpha. Cell 1998;93:693704.

9. Alberti S, Schuster G, Parini P et al: Hepatic cholesterol metabolism and resistance to dietary cholesterol in LXRbeta-deficient mice. J Clin Invest 2001;107:565-73.

10. Repa JJ, Liang G, Ou J et al: Regulation of mouse sterol regulatory element-binding protein-1c gene (SREBP-1c) by oxysterol receptors, LXRalpha and LXRbeta. Genes Dev 2000;14:2819-30.

11. Makishima M, Okamoto AY, Repa JJ et al: Identification of a nuclear receptor for bile acids. Science 1999;284:1362-5.

12. Wang $\mathrm{H}$, Chen $\mathrm{J}$, Hollister $\mathrm{K}$ et al: Endogenous bile acids are ligands for the nuclear receptor FXR/BAR. Mol Cell 1999;3:543-53.

13. Baker AR, McDonnell DP, Hughes $M$ et al: Cloning and expression of full-length cDNA encoding human vitamin D receptor. Proc Natl Acad Sci USA 1988;8 5:3294-8.

14. Kato $\mathrm{S}$ : The function of vitamin $\mathrm{D}$ receptor in vitamin D action. J Biochem 2000;127:71 7-22.

15. Rochel N, Wurtz JM, Mitschler A et al: The crystal structure of the nuclear receptor for vitamin D bound to its natural ligand. Mol Cell 2000;5:173-9.

16. Lala DS, Syka PM, Lazarchik SB et al: Activation of the orphan nuclear receptor steroidogenic factor 1 by oxysterols. Proc Natl Acad Sci USA 1997;94:4895-900.

17. Mellon SH, Bair SR: 25-Hydroxycholesterol is not a ligand for the orphan nuclear receptor steroidogenic factor-1 (SF-1). Endocrinology 1998;139:3026-9.

18. Lynch JP, Lala DS, Peluso JJ et al: Steroidogenic factor 1 , an orphan nuclear receptor, regulates the expression of the rat aromatase gene in gonadal tissues. Mol Endocrinol 1993;7:776-86.

19. Jump DB, Clarke SD: Regulation of gene expression by dietary fat. Annu Rev Nutr 1999;19:63-90.

20a. Göttlicher M, Widmark E, Li Q, Gustafsson JÅ. Fatty acids activate a chimera of the clofibric acid-activated receptor and the glucocorticoid receptor. Proc Natl Acad Sci 1992;89:4653-7.

20b. Xu HE, Lambert MH, Montana VG et al: Molecular recognition of fatty acids by peroxisome proliferator-activated receptors. Mol Cell 1999;3:397-403.

21. Issemann I, Prince RA, Tugwood JD, Green S: The peroxisome proliferator-activated receptor: retinoid $\mathrm{X}$ receptor heterodimer is activated by fatty acids and fibrate hypolipidaemic drugs. $\mathrm{J}$ Mol Endocrinol 1993;11:37-47.

22. Gonzalez FJ: Recent update on the PPAR alpha-null mouse. Biochimie 1997;79:139-44.

23. Forman BM, Chen J, Evans RM: Hypolipidemic drugs, polyunsaturated fatty acids, and eicosanoids are ligands 
for peroxisome proliferator-activated receptors $\alpha$ and $\delta$. Proc Natl Acad Sci USA 1997;94:4312-7.

24. Escher P, Braissant O, Basu-Modak $S$ et al: Rat PPARs: quantitative analysis in adult rat tissues and regulation in fasting and refeeding. Endocrinology 2001;142:4195-202.

25. Murphy GJ, Holder JC: PPAR-gamma agonists: therapeutic role in diabetes, inflammation and cancer. Trends Pharmacol Sci 2000;21:469-74.

26. Sood V, Colleran K, Burge MR: Thiazolidinediones: a comparative review of approved uses. Diabetes Technol Ther 2000;2:429-40.

27. Forman BM, Tontonoz P, Chen J et al: 15-Deoxy$\Delta 12,14$-prostaglandin $\mathrm{J} 2$ is a ligand for the adipocyte determination factor PPAR gamma. Cell 1995;83:80312.

28. Chawla A, Boisvert WA, Lee $\mathrm{CH}$ et al: A PPAR gamma-LXR-ABCA 1 pathway in macrophages is involved in cholesterol efflux and atherogenesis. Mol Cell 2001;7:161-71.

29. Hertz R, Magenheim J, Berman I, Bar TJ: Fatty acyl$\mathrm{CoA}$ thioesters are ligands of hepatic nuclear factor- $4 \alpha$. Nature 1998;392:512-6.

30. Ryffel GU: Mutations in the human genes encoding the transcription factors of the hepatocyte nuclear factor (HNF)1 and HNF4 families: functional and pathological consequences. J Mol Endocrinol 2001;27:1129.

31. Yamagata K, Furuta H, Oda $\mathrm{N}$ et al: Mutations in the hepatocyte nuclear factor- $4 \alpha$ gene in maturity-onset diabetes of the young (MODY1). Nature 1996; 384:458-60.

32. Lausen J, Thomas H, Lemm I et al: Naturally occurring mutations in the human HNF4alpha gene impair the function of the transcription factor to a varying degree. Nucl Acids Res 2000;28:430-7.

33. Shih DQ, Dansky HM, Fleisher M et al: Genotype/phenotype relationships in HNF-4alpha/MODY1: haploinsufficiency is associated with reduced apolipoprotein (AII), apolipoprotein (CIII), lipoprotein(a), and triglyceride levels. Diabetes 2000;49:832-7.

34. Andre E, Conquet F, Steinmayr M et al: Disruption of retinoid-related orphan receptor beta changes circadian behavior, causes retinal degeneration and leads to vacillans phenotype in mice. EMBO J 1998;17:3867-77.

35. Stehlin C, Wurtz JM, Steinmetz A et al: X-ray structure of the orphan nuclear receptor ROR beta ligand-binding domain in the active conformation. EMBO $\mathbf{J}$ 2001;20:5822-31.

36. Chambon P: A decade of molecular biology of retinoic acid receptors. FASEB J 1996;10:940-54.
37. Leblanc BP, Stunnenberg HG: 9-cis retinoic acid signaling: changing partners causes some excitement. Genes Dev 1995;9:1811-6.

38. Kitareewan S, Burka LT, Tomer KB et al: Phytol metabolites are circulating dietary factors that activate the nuclear receptor RXR. Mol Biol Cell 1996;7:115366.

39. Liu YL, Sennitt MV, Hislop DC et al: Retinoid X receptor agonists have anti-obesity effects and improve insulin sensitivity in Zucker fa/fa rats. Int $\mathrm{J}$ Obes Relat Metab Disord 2000;24:997-1004.

40. Kuiper GG, Enmark E, Pelto-Huikko $M$ et al: Cloning of a novel receptor expressed in rat prostate and ovary. Proc Natl Acad Sci USA 1996;93:5925-30.

41. Kuiper GG, Lemmen JG, Carlsson B et al: Interaction of estrogenic chemicals and phytoestrogens with estrogen receptor beta. Endocrinology 1998;139:4252-63.

42. Glazier MG, Bowman MA: A review of the evidence for the use of phytoestrogens as a replacement for traditional estrogen replacement therapy. Arch Intern Med 2001;161:1161-72.

43. Wynne-Edwards KE: Evolutionary biology of plant defenses against herbivory and their predictive implications for endocrine disruptor susceptibility in vertebrates. Environ Health Perspect 2001;109:443-8.

44. Lehmann JM, McKee DD, Watson MA et al: The human orphan nuclear receptor PXR is activated by compounds that regulate CYP3A4 gene expression and cause drug interactions. J Clin Invest 1998;102:101 6-23.

45. Moore JT, Kliewer SA: Use of the nuclear receptor PXR to predict drug interactions. Toxicology 2000; $153: 1-10$.

46. Moore LB, Goodwin B, Jones SA et al: St. John's wort induces hepatic drug metabolism through activation of the pregnane $\mathrm{X}$ receptor. Proc Natl Acad Sci USA 2000;97:7500-2.

47. Forman BM, Tzameli I, Choi HS et al: Androstane metabolites bind to and deactivate the nuclear receptor CAR-beta. Nature 1998;395:612-5.

48. Tzameli I, Pissios P, Schuetz EG, Moore DD: The xenobiotic compound 1,4-bis[2-(3,5-dichloropyridyloxy)]benzene is an agonist ligand for the nuclear receptor CAR. Mol Cell Biol 2000;20:2951-8.

49. Moore LB, Parks DJ, Jones SA et al: Orphan nuclear receptors constitutive androstane receptor and pregnane $\mathrm{X}$ receptor share xenobiotic and steroid ligands. $\mathrm{J}$ Biol Chem 2000;275:15122-7.

\footnotetext{
Jan-Åke Gustafsson

Departments of Biosciences and Medical Nutrition, Karolinska Institute, NOVUM, SE-14186 Huddinge, Sweden.

E-mail: jan-ake.gustafsson@csb.ki.se
} 